\title{
Differential equations arising from the generating function of general modified degenerate Euler numbers
}

Tae Kyun Kim ${ }^{1,2^{*}}$, Dae San Kim³ ${ }^{3}$ Hyuck In Kwon ${ }^{2}$ and Jong Jin Seo ${ }^{4}$

\section{"Correspondence:}

kimtk2015@gmail.com

'Department of Mathematics,

College of Science, Tianjin

Polytechnic University, Tianjin, 300387, China

${ }^{2}$ Department of Mathematics, Kwangwoon University, Seoul,

139-701, Republic of Korea

Full list of author information is

available at the end of the article

\begin{abstract}
In this paper, we introduce the general modified degenerate Euler numbers and study ordinary differential equations arising from the generating function of these numbers. In addition, we give some new explicit identities for the general modified degenerate Euler numbers arising from our differential equations.
\end{abstract}

MSC: 05A19; 11B37; 11B83; 34A34

Keywords: general modified degenerate Euler numbers; differential equations

\section{Introduction}

As is known, the Euler numbers are defined by the generating function

$$
\frac{2}{e^{t}+1}=\sum_{n=0}^{\infty} E_{n} \frac{t^{n}}{n !} \quad(\text { see }[1-9])
$$

Carlitz [2] considered the degenerate Euler numbers defined by the generating function

$$
\frac{2}{(1+\lambda t)^{\frac{1}{\lambda}}+1}=\sum_{n=0}^{\infty} \mathcal{E}_{n, \lambda} \frac{t^{n}}{n !}
$$

In [7], the modified degenerate Euler numbers, which are slightly different from Carlitz's degenerate Euler numbers, are defined by

$$
\frac{2}{(1+\lambda)^{\frac{t}{\lambda}}+1}=\sum_{n=0}^{\infty} \tilde{\mathcal{E}}_{n, \lambda} \frac{t^{n}}{n !}
$$

Note that $\lim _{\lambda \rightarrow 0} \tilde{\mathcal{E}}_{n, \lambda}=\lim _{\lambda \rightarrow 0} \mathcal{E}_{n, \lambda}=E_{n}(n \geq 0)$. Recently, Kim and Kim [6] studied nonlinear differential equations given by

$$
\left(\frac{d}{d t}\right)^{N}\left(\frac{1}{(1+\lambda t)^{\frac{1}{\lambda}}+1}\right)=\frac{(-1)^{N}}{(1+\lambda t)^{N}} \sum_{i=1}^{N+1} a_{i}(N, \lambda) F^{i},
$$

where $F=\frac{1}{(1+\lambda t)^{\frac{1}{\lambda}}+1}$.

(c) $2016 \mathrm{Kim}$ et al. This article is distributed under the terms of the Creative Commons Attribution 4.0 International License (http://creativecommons.org/licenses/by/4.0/), which permits unrestricted use, distribution, and reproduction in any medium, provided you give appropriate credit to the original author(s) and the source, provide a link to the Creative Commons license, and indicate if changes were made. 
Let $\alpha, a, b$ be nonzero real numbers. Then we consider the general modified degenerate Euler numbers as follows:

$$
\frac{2}{\alpha(1+\lambda)^{\frac{a t}{\lambda}}+b}=\sum_{n=0}^{\infty} \tilde{\mathcal{E}}_{n, \lambda}(\alpha \mid a, b) \frac{t^{n}}{n !} .
$$

From (1.5) we note that

$$
\begin{aligned}
\lim _{\lambda \rightarrow 0} \frac{2}{\alpha(1+\lambda)^{\frac{a t}{\lambda}}+b} & =\frac{2}{\alpha e^{a t}+b} \\
& =\frac{1}{b} \frac{2}{\frac{\alpha}{b} e^{a t}+1} \\
& =\frac{1}{b} \sum_{n=0}^{\infty} E_{n, \frac{\alpha}{b}} a^{n} \frac{t^{n}}{n !},
\end{aligned}
$$

where $E_{n, q}(n \geq 0)$ are the Apostol-Euler numbers given by the generating function

$$
\frac{2}{q e^{t}+1}=\sum_{n=0}^{\infty} E_{n, q} \frac{t^{n}}{n !} \quad(\text { see }[1,3])
$$

Thus, by (1.5) and (1.6) we get

$$
\frac{a^{n}}{b} E_{n, \frac{\alpha}{b}}=\lim _{\lambda \rightarrow 0} \tilde{\mathcal{E}}_{n, \lambda}(\alpha \mid a, b) \quad(n \geq 0) .
$$

Bayad and Kim [1] studied the following nonlinear differential equations related to Apostol-Euler numbers:

$$
F_{q}^{N}=\frac{1}{(N-1) !} \sum_{k=0}^{N} a_{k}(N) F_{q}^{(k-1)} \quad(N \in \mathbb{N}),
$$

where $F_{q}^{(k)}=\left(\frac{d}{d t}\right)^{k} F_{q}(t), F_{q}(t)=\frac{1}{q e^{t}+1}$.

In this paper, we study the ordinary differential equations associated with the generating function of general modified degenerate Euler numbers. In addition, we give some new and explicit formulas and identities for those numbers arising from our differential equations.

\section{Generalized modified degenerate Euler numbers}

For nonzero real numbers $\alpha, a, b$, let

$$
F=F(t)=\frac{1}{\alpha(1+\lambda)^{\frac{a t}{\lambda}}+b} .
$$

Then by (2.1) we get

$$
\begin{aligned}
F^{(1)} & =\frac{d F}{d t}(t) \\
& =\frac{(-1) \frac{a}{\lambda} \log (1+\lambda)}{\left(\alpha(1+\lambda)^{\frac{a t}{\lambda}}+b\right)^{2}}\left(\alpha(1+\lambda)^{\frac{\alpha t}{\lambda}}\right)
\end{aligned}
$$




$$
\begin{aligned}
& =\frac{(-1) \frac{a}{\lambda} \log (1+\lambda)}{\left(\alpha(1+\lambda)^{\frac{a t}{\lambda}}+b\right)^{2}}\left\{\alpha(1+\lambda)^{\frac{a t}{\lambda}}+b-b\right\} \\
& =(-1) \frac{a}{\lambda} \log (1+\lambda)\left(F-b F^{2}\right) .
\end{aligned}
$$

Thus, from (2.2) we have

$$
F^{(1)}=\frac{a}{\lambda} \log (1+\lambda)\left(b F^{2}-F\right) .
$$

From (2.3) we derive the following equation:

$$
\begin{aligned}
F^{(2)} & =\frac{d}{d t} F^{(1)} \\
& =\frac{a}{\lambda} \log (1+\lambda)\left\{2 b F F^{(1)}-F^{(1)}\right\} \\
& =\frac{a}{\lambda} \log (1+\lambda)(2 b F-1) F^{(1)} \\
& =\left(\frac{a}{\lambda} \log (1+\lambda)\right)^{2}(2 b F-1)\left(b F^{2}-F\right) \\
& =\left(\frac{a}{\lambda} \log (1+\lambda)\right)^{2}\left(2 b^{2} F^{3}-3 b F^{2}+F\right) .
\end{aligned}
$$

Continuing this process, we set

$$
\begin{aligned}
F^{(N)} & =\left(\frac{d}{d t}\right)^{N} F(t) \\
& =\left(\frac{a}{\lambda} \log (1+\lambda)\right) \sum_{k=1}^{N+1} a_{k}(N) b^{k-1} F^{k} .
\end{aligned}
$$

By taking the derivative of (2.5) with respect to $t$ we have

$$
\begin{aligned}
F^{(N+1)} & =\frac{d F^{(N)}}{d t} \\
& =\left(\frac{a}{\lambda} \log (1+\lambda)\right)^{N} \sum_{k=1}^{N+1} a_{k}(N) b^{k-1} k F^{k-1} F^{(1)} \\
& =\left(\frac{a}{\lambda} \log (1+\lambda)\right)^{N+1} \sum_{k=1}^{N+1}\left(k a_{k}(N) b^{k} F^{k+1}-a_{k}(N) b^{k-1} k F^{k}\right) \\
& =\left(\frac{a}{\lambda} \log (1+\lambda)\right)^{N+1}\left\{\sum_{k=2}^{N+2}(k-1) a_{k-1}(N) b^{k-1} F^{k}-\sum_{k=1}^{N+1} k a_{k}(N) b^{k-1} F^{k}\right\} .
\end{aligned}
$$

Replacing $N$ by $N+1$ in (2.5), we get

$$
F^{(N+1)}=\left(\frac{a}{\lambda} \log (1+\lambda)\right) \sum_{k=1}^{N+1} a_{k}(N+1) b^{k-1} F^{k} .
$$

Comparing the coefficients on both sides of (2.6) and (2.7), we obtain

$$
a_{1}(N+1)=-a_{1}(N) \text {. }
$$


Thus, by (2.8) we get

$$
a_{1}(N+1)=-a_{1}(N)=(-1)^{2} a_{1}(N-1)=\cdots=(-1)^{N} a_{1}(1) .
$$

From (2.5) we have

$$
\frac{a}{\lambda} \log (1+\lambda)\left\{b F^{2}-F\right\}=\frac{a}{\lambda} \log (1+\lambda)\left\{a_{1}(1) F+a_{2}(1) b F^{2}\right\}
$$

By (2.10) we get

$$
a_{1}(1)=-1 \quad \text { and } \quad a_{2}(1)=1 \text {. }
$$

Thus, from (2.9) and (2.11) we have

$$
a_{1}(N+1)=(-1)^{N} a_{1}(1)=(-1)^{N+1} .
$$

By (2.6) and (2.7) we see that

$$
\begin{aligned}
a_{N+2}(N+1)= & (N+1) a_{N+1}(N) \\
= & (N+1) N a_{N}(N-1) \\
= & (N+1) N(N-1) a_{N-1}(N-2) \\
& \vdots \\
= & (N+1) N(N-1) \cdots 2 a_{2}(1) \\
= & (N+1) ! .
\end{aligned}
$$

Thus, by (2.13) we have

$$
a_{N+2}(N+1)=(N+1) !
$$

For $2 \leq k \leq N+1$, by comparing the coefficients on both sides of (2.6) and (2.7) we have

$$
a_{k}(N+1)=(k-1) a_{k-1}(N)-k a_{k}(N) .
$$

Let $k=2$ in (2.15). Then we have

$$
\begin{aligned}
a_{2}(N+1) & =a_{1}(N)-2 a_{2}(N) \\
& =a_{1}(N)-2\left(a_{1}(N-1)-2 a_{2}(N-1)\right) \\
& =a_{1}(N)-2 a_{1}(N-1)+(-1)^{2} 2^{2} a_{2}(N-1) \\
& =a_{1}(N)-2 a_{1}(N-1)+(-1)^{2} 2^{2}\left\{a_{1}(N-2)-2 a_{2}(N-2)\right\} \\
& =a_{1}(N)-2 a_{1}(N-1)+(-1)^{2} 2^{2} a_{1}(N-2)+(-1)^{3} 2^{3} a_{2}(N-2)
\end{aligned}
$$




$$
\begin{aligned}
& =\sum_{k=0}^{N-1}(-1)^{k} a_{1}(N-k) 2^{k}+(-1)^{N} 2^{N} a_{2}(1) \\
& =\sum_{k=0}^{N}(-1)^{k} a_{1}(N-k) 2^{k} .
\end{aligned}
$$

For $k=3$ in (2.15), we have

$$
\begin{aligned}
a_{3}(N+1)= & 2 a_{2}(N)-3 a_{3}(N) \\
= & 2 a_{2}(N)-3\left\{2 a_{2}(N-1)-3 a_{3}(N-1)\right\} \\
= & 2 a_{2}(N)-3 \cdot 2 a_{2}(N-1)+(-1)^{2} 3^{2} a_{3}(N-1) \\
= & 2 a_{2}(N)-3 \cdot 2 a_{2}(N-1)+(-1)^{2} 3^{2}\left\{2 a_{2}(N-2)-3 a_{3}(N-2)\right\} \\
= & 2 a_{2}(N)-3 \cdot 2 a_{2}(N-1)+(-1)^{2} 3^{2} 2 a_{2}(N-2)+(-1)^{3} 3^{3} a_{3}(N-2) \\
& \vdots \\
= & 2 \sum_{k=0}^{N-2} a_{2}(N-k)(-1)^{k} 3^{k}+(-1)^{N-1} 3^{N-1} a_{3}(2) \\
= & 2 \sum_{k=0}^{N-1} a_{2}(N-k)(-1)^{k} 3^{k} .
\end{aligned}
$$

Continuing this process, we deduce

$$
a_{j}(N+1)=(j-1) \sum_{k=0}^{N-j+2} a_{j-1}(N-k)(-1)^{k} j^{k},
$$

where $2 \leq j \leq N+1$.

Now we give an explicit expression for $a_{j}(N+1)$ in (2.18). From (2.12) and (2.16) we can derive the following equation:

$$
\begin{aligned}
a_{2}(N+1) & =\sum_{k=0}^{N}(-1)^{k} a_{1}(N-k) 2^{k} \\
& =\sum_{k=0}^{N}(-1)^{k}(-1)^{N-k} 2^{k}=(-1)^{N} \sum_{k=0}^{N} 2^{k} .
\end{aligned}
$$

By (2.17) we get

$$
\begin{aligned}
a_{3}(N+1) & =2 \sum_{k_{2}=0}^{N-1} a_{2}\left(N-k_{2}\right)(-1)^{k_{2}} 3^{k_{2}} \\
& =2 \sum_{k_{2}=0}^{N-1}(-1)^{N-k_{2}-1} \sum_{k_{1}=0}^{N-k_{2}-1} 2^{k_{1}}(-1)^{k_{2}} 3^{k_{2}} \\
& =2(-1)^{N-1} \sum_{k_{2}=0}^{N-1} \sum_{k_{1}=0}^{N-1-k_{2}} 2^{k_{1}} 3^{k_{2}}
\end{aligned}
$$


Continuing this process, we deduce that, for $2 \leq j \leq N+1$,

$$
\begin{aligned}
a_{j}(N+1) & \\
= & (j-1) !(-1)^{N-j+2} \\
& \times \sum_{k_{j-1}=0}^{N-j+2} \sum_{k_{j-2}=0}^{N-j+2-k_{j-1}} \sum_{k_{j-3}=0}^{N-j+2-k_{j-1}-k_{j-2}} \cdots \sum_{k_{1}=0}^{N-j+2-k_{j-1} \cdots-k_{2}} j^{k_{j-1}}(j-1)^{k_{j-2}} \cdots 3^{k_{2}} 2^{k_{1}} .
\end{aligned}
$$

Therefore, by (2.5) and (2.21) we obtain the following theorem.

Theorem 1 Let $\alpha, a, b$ be nonzero real numbers. The family of nonlinear differential equations

$$
F^{(N)}=\left(\frac{a}{\lambda} \log (1+\lambda)\right)^{N} \sum_{k=1}^{N+1} a_{k}(N) b^{k-1} F^{k}
$$

has a solution $F=F(t)=\frac{1}{\alpha(1+\lambda)^{\frac{a t}{\lambda}}+b}$, where $a_{1}(N)=(-1)^{N}$, and

$$
\begin{aligned}
a_{j}(N)= & (j-1) !(-1)^{N-j+1} \\
& \times \sum_{k_{j-1}=0}^{N-j+1} \sum_{k_{j-2}=0}^{N-j+1-k_{j-1}} \cdots \sum_{k_{1}=0}^{N-j+1-k_{j-1}-\cdots-k_{2}} j^{k_{j-1}}(j-1)^{k_{j-2}} \cdots 3^{k_{2}} 2^{k_{1}}
\end{aligned}
$$

for $2 \leq j \leq N+1$.

Now we define the general modified degenerate Euler numbers given by the generating function

$$
\frac{2}{\alpha(1+\lambda)^{\frac{a t}{\lambda}}+b}=\sum_{n=0}^{\infty} \tilde{\mathcal{E}}_{n, \lambda}(\alpha ; a, b) \frac{t^{n}}{n !} .
$$

Note that $\tilde{\mathcal{E}}_{n, \lambda}(1 ; 1,1)$ are the modified degenerate Euler numbers given by

$$
\frac{2}{(1+\lambda)^{\frac{t}{\lambda}}+1}=\sum_{n=0}^{\infty} \tilde{\mathcal{E}}_{n, \lambda} \frac{t^{n}}{n !}
$$

Now we observe that

$$
\begin{aligned}
F^{(N)} & =\frac{1}{2}\left(\frac{d}{d t}\right)^{N}\left(\frac{2}{\alpha(1+\lambda)^{\frac{a t}{\lambda}}+b}\right) \\
& =\frac{1}{2} \sum_{n=0}^{\infty} \frac{\tilde{\mathcal{E}}_{n, \lambda}(\alpha ; a, b)}{n !}\left(\frac{d}{d t}\right)^{N} t^{n} \\
& =\frac{1}{2} \sum_{n=0}^{\infty} \tilde{\mathcal{E}}_{n+N, \lambda}(\alpha ; a, b) \frac{t^{n}}{n !} .
\end{aligned}
$$


For $r \in \mathbb{N}$, the higher-order general modified degenerate Euler numbers are defined by the generating function

$$
\left(\frac{2}{\alpha(1+\lambda)^{\frac{a t}{\lambda}}+b}\right)^{r}=\sum_{n=0}^{\infty} \tilde{\mathcal{E}}_{n, \lambda}^{(r)}(\alpha ; a, b) \frac{t^{n}}{n !}
$$

Therefore, by Theorem 1, (2.23), and (2.21) we obtain the following theorem.

Theorem 2 Let $\alpha, a, b$ be nonzero real numbers. For $n \geq 0$, we have

$$
\tilde{\mathcal{E}}_{n+N}(\alpha ; a, b)=\left(\frac{a}{\lambda} \log (1+\lambda)\right)^{N} \sum_{k=1}^{N+1} a_{k}(N) b^{k-1} 2^{1-k} \tilde{\mathcal{E}}_{n, \lambda}^{(k)}(\alpha ; a, b)
$$

where $a_{1}(N)=(-1)^{N}$, and, for $2 \leq j \leq N+1$,

$$
\begin{aligned}
a_{j}(N)= & (j-1) !(-1)^{N-j+1} \\
& \times \sum_{k_{j-1}=0}^{N-j+1} \sum_{k_{j-2}=0}^{N-j+1-k_{j-1}} \cdots \sum_{k_{1}=0}^{N-j+1-k_{j-1}-\cdots-k_{2}} j^{k_{j-1}}(j-1)^{k_{j-2}} \cdots 3^{k_{2}} 2^{k_{1}} .
\end{aligned}
$$

\section{Competing interests}

The authors declare that they have no competing interests.

\section{Authors' contributions}

All authors contributed equally to this work. All authors read and approved the final manuscript.

\section{Author details}

'Department of Mathematics, College of Science, Tianjin Polytechnic University, Tianjin, 300387, China. ${ }^{2}$ Department of Mathematics, Kwangwoon University, Seoul, 139-701, Republic of Korea. ${ }^{3}$ Department of Mathematics, Sogang University, Seoul, 121-742, Republic of Korea. ${ }^{4}$ Department of Applied Mathematics, Pukyong National University, Busan, Republic of Korea.

\section{Acknowledgements}

The first author is appointed as a chair professor at Tianjin Polytechnic University, Tianjin City, China, from August 2015 to August 2019. We would like to thank the referee for his detailed suggestions that helped to improve the original manuscript.

Received: 11 April 2016 Accepted: 9 May 2016 Published online: 11 May 2016

\section{References}

1. Bayad, A, Kim, T: Higher recurrences for Apostol-Bernoulli-Euler numbers. Russ. J. Math. Phys. 19(1), 1-10 (2012). MR2892600

2. Carlitz, L: Degenerate Stirling, Bernoulli and Eulerian numbers. Util. Math. 15, 51-88 (1979). MR531621

3. Ding, D, Yang, J: Some identities related to the Apostol-Euler and Apostol-Bernoulli polynomials. Adv. Stud. Contemp. Math. (Kyungshang) 20(1), 7-21 (2010). MR2597988

4. Dolgy, DV, Kim, T, Kwon, HI, Seo, JJ: On the modified degenerate Bernoulli polynomials. Adv. Stud. Contemp. Math. (Kyungshang) 26(1), 1-9 (2016)

5. Gaboury, S, Tremblay, R, Fugère, B-J: Some explicit formulas for certain new classes of Bernoulli, Euler and Genocchi polynomials. Proc. Jangjeon Math. Soc. 17(1), 115-123 (2014). MR3184467

6. Kim, T, Kim, DS: Identities involving degenerate Euler numbers and polynomials arising from non-linear differential equations. J. Nonlinear Sci. Appl. 9, 2086-2098 (2016)

7. Kwon, HI, Kim, T, Seo, JJ: Modified degenerate Euler polynomials. Adv. Stud. Contemp. Math. (Kyungshang) 26(1), 203-209 (2016)

8. Rim, S-H, Jeong, J: On the modified $q$-Euler numbers of higher order with weight. Adv. Stud. Contemp. Math. (Kyungshang) 22(1), 93-98 (2012). MR2931608

9. Zhang, Z, Yang, H: Some closed formulas for generalized Bernoulli-Euler numbers and polynomials. Proc. Jangjeon Math. Soc. 11(2), 191-198 (2008). MR2482602 